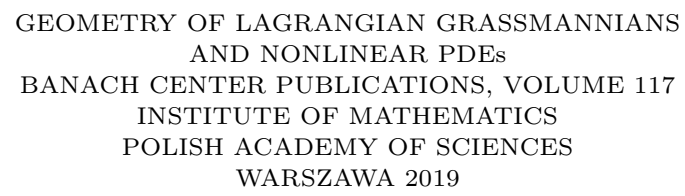

\title{
INTRODUCTION TO EXTERIOR DIFFERENTIAL SYSTEMS
}

\author{
BENJAMIN MCKAY \\ University College Cork, Cork, Ireland \\ E-mail:b.mckay@ucc.ie
}

\begin{abstract}
These lectures explain how to apply the Cartan-Kähler theorem to problems in differential geometry. We want to decide if there are submanifolds of a given dimension inside a given manifold on which given differential forms vanish. The Cartan-Kähler theorem gives a linear algebra test: if the test passes, such submanifolds exist. I will not give a proof or give the most general statement of the theorem, as it is difficult to state precisely.
\end{abstract}

1. Expressing differential equations using differential forms. Take a differential equation of second order $0=f\left(x, u, u_{x}, u_{x x}\right)$. To write it as a first order system, add a new variable $p$ to represent $u_{x}$, and a new equation:

$$
\begin{gathered}
u_{x}=p, \\
0=f\left(x, u, p, p_{x}\right) .
\end{gathered}
$$

It is easy to generalize this to any number of variables and equations of any order: reduce any system of differential equation to a first order system.

To express a first order differential equation $0=f\left(x, u, u_{x}\right)$, add a variable $p$ to represent the derivative $u_{x}$, let $\vartheta=d u-p d x$ on the manifold

$$
M=\{(x, u, p) \mid 0=f(x, u, p)\}
$$

(assuming it is a manifold). A submanifold of $M$ of suitable dimension on which $0=\vartheta$ and $0 \neq d x$ is locally the graph of a solution. It is easy to generalize this to any number of variables and any number of equations of any order.

2010 Mathematics Subject Classification: 58A15.

Key words and phrases: Exterior Differential Systems.

Thanks to Daniel Piker for the use of his images of triply orthogonal webs.

The paper is in final form and no version of it will be published elsewhere. 
2. The Cartan-Kähler theorem. An integral manifold of a collection of differential forms is a submanifold on which the forms vanish. An exterior differential system is an ideal $\mathcal{I} \subset \Omega^{*}$ of smooth differential forms on a manifold $M$, closed under exterior derivative, which splits into a direct sum

$$
\mathcal{I}=\mathcal{I}^{0} \oplus \mathcal{I}^{1} \oplus \ldots \oplus \mathcal{I}^{n}
$$

of forms of various degrees: $\mathcal{I}^{p}:=\mathcal{I} \cap \Omega^{p}$. Any collection of differential forms has the same integral manifolds as the exterior differential system it generates. An exterior differential system is analytic if it is locally generated by real analytic differential forms.

Some trivial examples: the exterior differential system generated by

1. 0 ,

2. $\Omega^{*}$,

3. the pullbacks of all forms via a submersion,

4. $d x^{1} \wedge d y^{1}+d x^{2} \wedge d y^{2}$ in $\mathbb{R}^{4}$,

5. $d y-z d x$ on $\mathbb{R}^{3}$.

Problem 1. What are the integral manifolds of our trivial examples?

The elements of $\mathcal{I}^{0}$ are 0 -forms, i.e. functions. All $\mathcal{I}$-integral manifolds lie in the zero locus of these functions. Replace our manifold $M$ by that zero locus (which might not be a manifold, a technical detail we will ignore); henceforth we add to the definition of exterior differential system the requirement that $\mathcal{I}^{0}=0$.

An integral element at a point $m \in M$ of an exterior differential system $\mathcal{I}$ is a linear subspace $E \subset T_{m} M$ on which all forms in $\mathcal{I}$ vanish. Every tangent space of an integral manifold is an integral element, but some integral elements of some exterior differential systems do not arise as tangent spaces of integral manifolds.

Problem 2. What are the integral elements of our trivial examples?

The polar equations of an integral element $E$ are the linear functions

$$
w \in T_{m} M \mapsto \vartheta\left(w, e_{1}, e_{2}, \ldots, e_{k}\right)
$$

where $\vartheta \in \mathcal{I}^{k+1}$ and $e_{1}, e_{2}, \ldots, e_{k} \in E$. They vanish on a vector $w$ just when the span of $\{w\} \cup E$ is an integral element. If an integral element $E$ is contained in another one, $E \subset F$, then all polar equations of $E$ occur among those of $F$ : larger integral elements have more (or at least the same) polar equations.

Problem 3. What are the polar equations of the integral elements of our trivial examples?

A partial flag $E_{\bullet}$ is a sequence of nested linear subspaces $E_{0} \subset E_{1} \subset E_{2} \subset \ldots \subset E_{p}$ in a vector space. The increments of a partial flag are the integers measuring how the dimensions increase:

$$
\begin{aligned}
& \operatorname{dim} E_{0}, \\
& \operatorname{dim} E_{1}-\operatorname{dim} E_{0}, \\
& \operatorname{dim} E_{2}-\operatorname{dim} E_{1}, \\
& \quad \vdots \\
& \operatorname{dim} E_{p}-\operatorname{dim} E_{p-1} .
\end{aligned}
$$


A flag is a partial flag

$$
E_{0} \subset E_{1} \subset E_{2} \subset \ldots \subset E_{p}
$$

for which $\operatorname{dim} E_{i}=i$. Danger: most authors require that a flag have subspaces of all dimensions; we do not: we only require that the subspaces have all dimensions $0,1,2, \ldots, p$ up to some dimension $p$. In particular, the increments of any flag are $0,1,1, \ldots, 1$.

The polar equations of a flag $E_{\bullet}$ of integral elements form a partial flag in the cotangent space. The characters $s_{0}, s_{1}, \ldots, s_{p}$ of $E_{\bullet}$ are the increments of its polar equations, i.e. the numbers of linearly independent polar equations added at each increment in the flag.

Problem 4. What are the characters of the integral flags of our trivial examples?

The rank $p$ Grassmann bundle of a manifold $M$ is the set of all $p$-dimensional linear subspaces of tangent spaces of $M$.

Problem 5. Recall how charts are defined on the Grassmann bundle. Prove that the Grassmann bundle is a fiber bundle over the underlying manifold.

The integral elements of an exterior differential system form a subset of the Grassmann bundle. Let us inquire whether this subset is a submanifold of the Grassmann bundle; if so, let us predict its dimension. We say that a flag of integral elements predicts the dimension $\operatorname{dim} M+s_{1}+2 s_{2}+\ldots+p s_{p}$; an integral element predicts the dimension predicted by the generic flag inside it.

THEOREM 1 (Cartan's bound). Every integral element predicts the dimension of a submanifold of the Grassmann bundle containing all nearby integral elements.

An integral element $E$ correctly predicts dimension if the integral elements near $E$ form a manifold of dimension predicted by $E$. An integral element which correctly predicts dimension is involutive.

THEOREM 2 (Cartan-Kähler). There is an integral manifold tangent to every involutive integral element of any analytic exterior differential system.

If an integral element is involutive, then all nearby integral elements are too, as the nonzero polar equations will remain nonzero. An exterior differential system is involutive if its generic maximal dimensional integral element is involutive.

Problem 6. The Frobenius theorem in this language: on a manifold $M$ of dimension $p+q$, take an exterior differential system $\mathcal{I}$ locally generated by $q$ linearly independent 1-forms together with all differential forms of degree more than $p: \mathcal{I}^{k}=\Omega^{k}$ for $k>p$. Prove that $\mathcal{I}$ is involutive if and only if every 2 -form in $\mathcal{I}$ is a sum of terms of the form $\xi \wedge \vartheta$ where $\vartheta$ is a 1 -form in $\mathcal{I}$. Prove that this occurs just when the $p$-dimensional $\mathcal{I}$-integral manifolds form the leaves of a foliation $F$ of $M$. Prove that then $\mathcal{I}^{1}$ consists precisely of the 1 -forms vanishing on the leaves of $F$.

\section{Example: Lagrangian submanifolds. Let}

$$
\vartheta:=d x^{1} \wedge d y^{1}+d x^{2} \wedge d y^{2}+\ldots+d x^{n} \wedge d y^{n} .
$$

Let $\mathcal{I}$ be the exterior differential system generated by $\vartheta$ on $M:=\mathbb{R}^{2 n}$. The integral manifolds of $\mathcal{I}$ are called Lagrangian manifolds. Let us employ the Cartan-Kähler theorem 
to prove the existence of Lagrangian submanifolds of complex Euclidean space. Writing spans of vectors in angle brackets,

\begin{tabular}{lll}
\hline Flag & Polar equations & Characters \\
\hline$E_{0}=\{0\}$ & $\{0\}$ & $s_{0}=0$ \\
$E_{1}=\left\langle\partial_{x^{1}}\right\rangle$ & $\left\langle d y^{1}\right\rangle$ & $s_{1}=1$ \\
$\quad \vdots$ & $\vdots$ & $\vdots$ \\
$E_{n}=\left\langle\partial_{x^{1}}, \partial_{x^{2}}, \ldots, \partial_{x^{n}}\right\rangle$ & $\left\langle d y^{1}, d y^{2}, \ldots, d y^{n}\right\rangle$ & $s_{n}=1$ \\
\hline
\end{tabular}

The flag predicts

$$
\operatorname{dim} M+s_{1}+2 s_{2}+\ldots+n s_{n}=2 n+1+2+\ldots+n .
$$

The nearby integral elements at a given point of $M$ are parameterized by $d y=a d x$, which we plug in to $\vartheta=0$ to see that $a$ can be any symmetric matrix. So the space of integral elements has dimension

$$
\operatorname{dim} M+\frac{n(n+1)}{2}=2 n+\frac{n(n+1)}{2},
$$

correctly predicted. Therefore the Cartan-Kähler theorem proves the existence of Lagrangian submanifolds of complex Euclidean space, one (at least) through each point, tangent to each subspace $d y=a d x$, at least for any symmetric matrix $a$ close to 0 .

Problem 7. On a complex manifold $M$, take a Kähler form $\vartheta$ and a holomorphic volume form $\Psi$, i.e. closed forms expressed in local complex coordinates as

$$
\begin{aligned}
\vartheta & =\frac{\sqrt{-1}}{2} g_{\mu \bar{\nu}} d z^{\mu} \wedge d z^{\bar{\nu}}, \\
\Psi & =f(z) d z^{1} \wedge d z^{2} \wedge \ldots \wedge d z^{n},
\end{aligned}
$$

with $f(z)$ a holomorphic function and $g_{\mu \bar{\nu}}$ a positive definite self-adjoint complex matrix of functions. Prove the existence of special Lagrangian manifolds, i.e. integral manifolds of the exterior differential system generated by the pair of $\vartheta$ and the imaginary part of $\Psi$.

4. The last character. In applying the Cartan-Kähler theorem, we are always looking for submanifolds of a particular dimension $p$. For simplicity, we can add the hypothesis that our exterior differential system contains all differential forms of degree $p+1$ and higher. In particular, the $p$-dimensional integral elements are maximal dimensional integral elements. The polar equations of any maximal dimensional integral element $E_{p}$ cut out precisely $E_{p}$, i.e. there are $\operatorname{dim} M-p$ independent polar equations on $E_{p}$. We encounter $s_{0}, s_{1}, \ldots, s_{p}$ polar equations at each increment, so the number of independent polar equations is $s_{0}+s_{1}+\ldots+s_{p}$. Our hypothesis helps us to calculate $s_{p}$ from the other characters:

$$
s_{0}+s_{1}+\ldots+s_{p-1}+s_{p}=\operatorname{dim} M-p .
$$

For even greater simplicity, we take this as a definition for the final character $s_{p}$, throwing out the previous definition. Now we can ignore any differential forms of degree more than $p$ when we test Cartan's bound. 
5. Example: harmonic functions. We will prove the existence of harmonic functions on the plane with given value and first derivatives at a given point. On $M=\mathbb{R}_{x, y, u, u_{x}, u_{y}}^{5}$, let $\mathcal{I}$ be the exterior differential system generated by

$$
\begin{gathered}
\vartheta:=d u-u_{x} d x-u_{y} d y, \\
\Theta:=d u_{x} \wedge d y-d u_{y} \wedge d x .
\end{gathered}
$$

Note that

$$
d \vartheta=-d u_{x} \wedge d x-d u_{y} \wedge d y
$$

also belongs to $\mathcal{I}$ because any exterior differential system is closed under exterior derivative. An integral surface $X \subset M$ on which $0 \neq d x \wedge d y$ is locally the graph of a harmonic function $u=u(x, y)$ and its derivatives $u_{x}=\frac{\partial u}{\partial x}, u_{x}=\frac{\partial u}{\partial x}$.

Each integral plane $E_{2}$ (i.e. integral element of dimension two) on which $d x \wedge d y \neq 0$ is given by equations

$$
\begin{aligned}
& d u_{x}=u_{x x} d x+u_{x y} d y, \\
& d u_{y}=u_{y x} d x+u_{y y} d y
\end{aligned}
$$

for a unique choice of four constants $u_{x x}, u_{x y}, u_{y x}, u_{y y}$ subject to the two equations $u_{x y}=$ $u_{y x}$ and $0=u_{x x}+u_{y y}$. Hence integral planes at each point have dimension two. The space of integral planes has dimension $\operatorname{dim} M+2=5+2=7$.

Each vector inside that integral plane has the form

$$
v=\left(\dot{x}, \dot{y}, u_{x} \dot{x}+u_{y} \dot{y}, u_{x x} \dot{x}+u_{x y} \dot{y}, u_{y x} \dot{x}+u_{y y} \dot{y}\right) .
$$

Each integral line $E_{1}$ is the span $E_{1}=\langle v\rangle$ of a nonzero such vector. Compute

$$
v\lrcorner\left(\begin{array}{c}
d \vartheta \\
\Theta
\end{array}\right)=\left(\begin{array}{l}
\dot{x} d u_{x}+\dot{y} d u_{y}-\left(u_{x x} \dot{x}+u_{x y} \dot{y}\right) d x-\left(u_{y x} \dot{x}+u_{y y} \dot{y}\right) d y \\
\dot{y} d u_{x}-\dot{x} d u_{y}+\left(u_{x x} \dot{x}+u_{x y} \dot{y}\right) d y-\left(u_{y x} \dot{x}+u_{y y} \dot{y}\right) d x
\end{array}\right) .
$$

and

\begin{tabular}{lll}
\hline Flag & Polar equations & Characters \\
\hline$E_{0}=\{0\}$ & $\langle\vartheta\rangle$ & $s_{0}=1$ \\
$E_{1}=\langle v\rangle$ & $\langle\vartheta, v\lrcorner d \vartheta, v\lrcorner \Theta\rangle$ & $s_{1}=2$ \\
\hline
\end{tabular}

Since we are only interested in finding integral surfaces, we compute the final character from

$$
s_{0}+s_{1}+s_{2}=\operatorname{dim} M-2 .
$$

The Cartan characters are $\left(s_{0}, s_{1}, s_{2}\right)=(1,2,0)$ with predicted dimension $\operatorname{dim} M+s_{1}+$ $2 s_{2}=5+2+2 \cdot 0=7$ : involution. We see that harmonic functions exist near any point of the plane, with prescribed value and first derivatives at that point.

6. Generality of integral manifolds. The proof of the Cartan-Kähler theorem (which we will not give) constructs integral manifolds inductively, starting with a point, then building an integral curve, and so on. The choice of the initial data at each inductive stage consists of $s_{0}$ constants, $s_{1}$ functions of one variable, and so on. Different choices of this initial data give rise to different integral manifolds in the final stage. In this sense, the integral manifolds depend on $s_{0}$ constants, and so on. 
If one describes some family of submanifolds in terms of the integral manifolds of an exterior differential system, someone else might find a different description of the same submanifolds in terms of integral manifolds of a different exterior differential system, with different Cartan characters.

For example, any smooth function $y=f(x)$ of one variable is equivalent information to having a constant $f(0)$ and a function $y^{\prime}=f^{\prime}(x)$ of one variable.

For example, immersed plane curves are the integral curves of $\mathcal{I}=0$ on $M=\mathbb{R}^{2}$. Check that any integral flag $E_{0}=\{0\}, E_{1}=\langle v\rangle$ has $\left(s_{0}, s_{1}\right)=(0,1)$. But immersed plane curves are also the integral curves of the ideal $\mathcal{I}$ generated by

$$
\vartheta:=\sin \phi d x-\cos \phi d y
$$

on $M:=\mathbb{R}_{x, y}^{2} \times S_{\phi}^{1}$. Here $\left(s_{0}, s_{1}\right)=(1,1)$. The last nonzero character does not change. In general, we cannot expect all of the Cartan characters to stay the same for different descriptions of various submanifolds, but we can expect the last nonzero Cartan character to stay the same.

Lagrangian submanifolds of $\mathbb{C}^{n}$ depend on one function of $n$ variables. This count is correct: those which are graphs $y=y(x)$ are precisely of the form

$$
y=\frac{\partial S}{\partial x}
$$

for some potential function $S(x)$, unique up to adding a real constant. On the other hand, the proof of the Cartan-Kähler theorem builds up each Lagrangian manifold from a choice of one function of one variable, one function of two variables, and so on. Similarly, harmonic functions depend on two functions of one variable. Summing up: we "trust" the last nonzero Cartan-Kähler $s_{p}$ to tell us the generality of the integral manifolds: they depend on $s_{p}$ functions of $p$ variables, but we do not "trust" $s_{0}, s_{1}, \ldots, s_{p-1}$.

7. Example: triply orthogonal webs. On a three-dimensional Riemannian manifold $X$, a triply orthogonal web is a triple of foliations whose leaves are pairwise perpendicular. We will see that these exist, locally, depending on three functions of two variables. Each leaf is perpendicular to a unique smooth unit length 1 -form $\eta_{i}$, up to \pm , which satisfies $0=\eta_{i} \wedge d \eta_{i}$, by the Frobenius theorem. Let $M$ be the set of all orthonormal bases of the tangent spaces of $X$, with obvious bundle map $x: M \rightarrow X$, so that each point of $M$ has the form $m=\left(x, e_{1}, e_{2}, e_{3}\right)$ for some $x \in X$ and orthonormal basis $e_{1}, e_{2}, e_{3}$ of $T_{x} X$. The soldering 1-forms $\omega_{1}, \omega_{2}, \omega_{3}$ on $M$ are defined by

$$
v\lrcorner \omega_{i}=\left\langle e_{i}, x_{*} v\right\rangle .
$$

Note: they are 1 -forms on $M$, not on $X$. Let

$$
\omega=\left(\begin{array}{l}
\omega_{1} \\
\omega_{2} \\
\omega_{3}
\end{array}\right) .
$$

Define cross product $\alpha \times \beta$ on $\mathbb{R}^{3}$-valued 1 -forms by

$$
\alpha \times \beta(u, v)=\alpha(u) \times \beta(v)-\alpha(v) \times \beta(u) .
$$



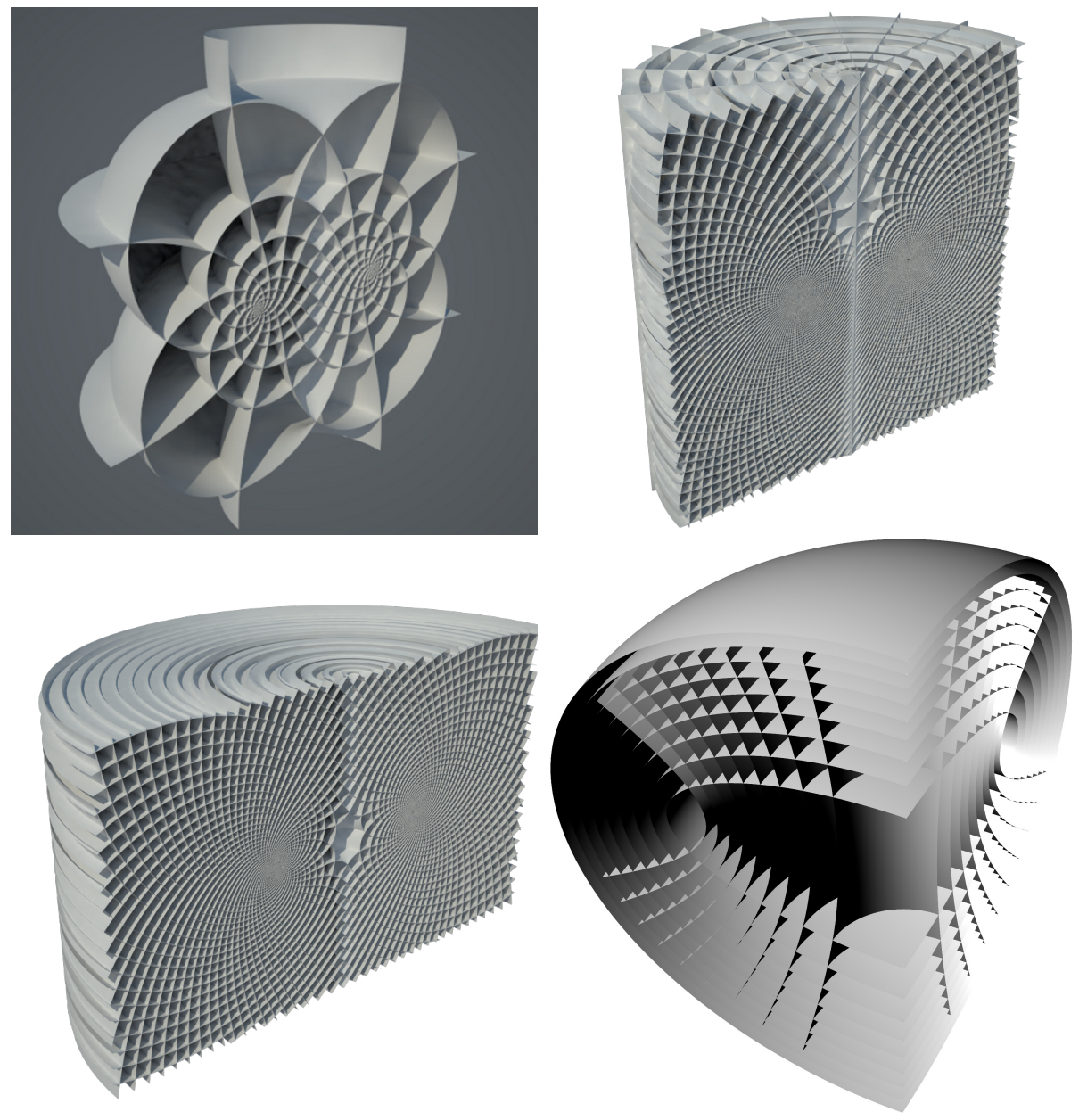

Images (a), (b), (c): Daniel Piker, 2015

By the fundamental lemma of Riemannian geometry, there is a unique $\mathbb{R}^{3}$-valued 1-form $\gamma$ for which $d \omega=\frac{1}{2} \gamma \times \omega$. Our triply orthogonal web is precisely a section $X \rightarrow M$ of the bundle map $M \rightarrow X$ on which $0=\omega_{i} \wedge d \omega_{i}$ for all $i$, hence an integral 3-manifold of the exterior differential system $\mathcal{I}$ on $M$ generated by the 3 -forms

$$
\omega_{1} \wedge d \omega_{1}, \omega_{2} \wedge d \omega_{2}, \omega_{3} \wedge d \omega_{3}
$$

Using the equations above, $\mathcal{I}$ is also generated by

$$
\gamma_{3} \wedge \omega_{1} \wedge \omega_{2}, \gamma_{1} \wedge \omega_{2} \wedge \omega_{3}, \gamma_{2} \wedge \omega_{3} \wedge \omega_{1}
$$

The three-dimensional $\mathcal{I}$-integral manifolds on which

$$
0 \neq \omega_{1} \wedge \omega_{2} \wedge \omega_{3}
$$

are locally precisely the triply orthogonal webs. The three-dimensional integral elements on which

$$
0 \neq \omega_{1} \wedge \omega_{2} \wedge \omega_{3}
$$


are precisely given by

$$
\gamma=\left(\begin{array}{ccc}
0 & p_{12} & p_{13} \\
p_{21} & 0 & p_{23} \\
p_{31} & p_{32} & 0
\end{array}\right) \omega
$$

for any $p_{i j}$, hence $6+6=12$ dimensions of integral elements. Since the system is generated by 3 -forms, on any integral flag in this integral element, $0=s_{0}=s_{1}$. Count out $\left(s_{0}, s_{1}, s_{2}, s_{3}\right)=(0,0,3,0)$, predicting 12 dimensions of integral elements: involution.

We conclude: for any orthonormal basis at a point of any real analytic Riemannian 3-manifold, there are infinitely many real analytic triply orthogonal webs, depending on three functions of two variables, defined near that point, with the tangent spaces of the leaves perpendicular to those basis vectors.

8. Example: isometric immersion. Take a surface $P$ with a Riemannian metric. Naturally we are curious if there is an isometric immersion $f: P \rightarrow \mathbb{R}^{3}$, i.e. a smooth map preserving the lengths of all curves on $P$. For example, these surfaces
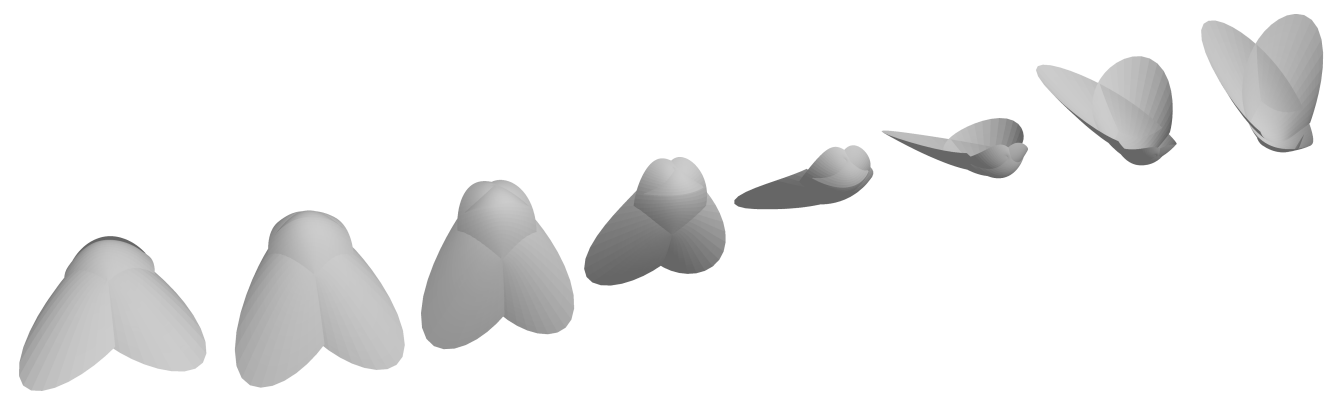

are isometric immersions of a piece of this paraboloid

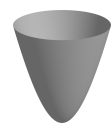

More generally, take a Riemannian manifold $\bar{P}$ of dimension three. We ask if there is an isometric immersion $f: P \rightarrow \bar{P}$.

On the orthonormal frame bundle $F P$, denote the soldering forms as $\omega=\omega_{1}+i \omega_{2}$. By the fundamental lemma of Riemannian geometry there is a unique 1-form (the connection 1-form) $\gamma$ so that $d \omega=i \gamma \wedge \omega$ and $d \gamma=(i / 2) K \omega \wedge \bar{\omega}$. As above, on $F \bar{P}$ there is a soldering 1 -form $\bar{\omega}$ and a connection 1 -form $\bar{\gamma}$ so that $d \bar{\omega}=\frac{1}{2} \bar{\gamma} \times \bar{\omega}$. This ensures that

$$
d \bar{\gamma}=\frac{1}{2} \bar{\gamma} \times \bar{\gamma}+\frac{1}{2}\left(\frac{s}{2}-R\right) \bar{\omega} \times \bar{\omega} .
$$

with Ricci curvature $R_{i j}=R_{j i}$ and scalar curvature $s=R_{i i}$.

If there is an isometric immersion $f: P \rightarrow \bar{P}$, then let $X:=X_{f} \subset M:=F P \times F \bar{P}$ be its adapted frame bundle, i.e. the set of all tuples

$$
\left(p, e_{1}, e_{2}, \bar{p}, \bar{e}_{1}, \bar{e}_{2}, \bar{e}_{3}\right)
$$

where $p \in P$ with orthonormal frame $e_{1}, e_{2} \in T_{p} P$ and $\bar{p} \in \bar{P}$ with orthonormal frames $\bar{e}_{1}, \bar{e}_{2}, \bar{e}_{3} \in T_{\bar{p}} \bar{P}$, so that $f_{*} e_{1}=\bar{e}_{1}$ and $f_{*} e_{2}=\bar{e}_{2}$. Let $\mathcal{I}$ be the exterior differential system 
on $M$ generated by the 1 -forms

$$
\left(\begin{array}{l}
\vartheta_{1} \\
\vartheta_{2} \\
\vartheta_{3}
\end{array}\right):=\left(\begin{array}{c}
\bar{\omega}_{1}-\omega_{1} \\
\bar{\omega}_{2}-\omega_{2} \\
\bar{\omega}_{3}
\end{array}\right)
$$

Along $X$, all of these 1 -forms vanish, while the 1 -forms $\omega_{1}, \omega_{2}, \gamma$ remain linearly independent. Conversely, we will eventually prove that all $\mathcal{I}$-integral manifolds on which $\omega_{1}, \omega_{2}, \gamma$ are linearly independent are locally frame bundles of isometric immersions. For the moment, we concentrate on asking whether we can apply the Cartan-Kähler theorem.

Compute:

$$
d\left(\begin{array}{l}
\vartheta_{1} \\
\vartheta_{2} \\
\vartheta_{3}
\end{array}\right)=-\left(\begin{array}{ccc}
0 & \bar{\gamma}_{3}-\gamma & 0 \\
-\left(\bar{\gamma}_{3}-\gamma\right) & 0 & 0 \\
\bar{\gamma}_{2} & -\bar{\gamma}_{1} & 0
\end{array}\right) \wedge\left(\begin{array}{c}
\omega_{1} \\
\omega_{2} \\
\gamma
\end{array}\right) \bmod \vartheta_{1}, \vartheta_{2}, \vartheta_{3} .
$$

We count $s_{1}=2, s_{2}=1, s_{3}=0$. Each three-dimensional integral element has $\bar{\omega}=\omega$, so is determined by the linear equations giving $\bar{\gamma}_{1}, \bar{\gamma}_{2}, \bar{\gamma}_{3}$ in terms of $\omega_{1}, \omega_{2}, \gamma$ on which $d \vartheta=0$ :

$$
\left(\begin{array}{c}
\bar{\gamma}_{1} \\
\bar{\gamma}_{2} \\
\bar{\gamma}_{3}-\gamma
\end{array}\right)=\left(\begin{array}{cc}
a & b \\
c & -a \\
0 & 0
\end{array}\right)\left(\begin{array}{l}
\omega_{1} \\
\omega_{2}
\end{array}\right)
$$

Therefore there is a three-dimensional space of integral elements at each point. But $s_{1}+2 s_{2}=4>3$ : no integral element correctly predicts dimension, so we cannot apply the Cartan-Kähler theorem.

What to do? On every integral element, we said that

$$
\left(\begin{array}{c}
\bar{\gamma}_{1} \\
\bar{\gamma}_{2} \\
\bar{\gamma}_{3}-\gamma
\end{array}\right)=\left(\begin{array}{cc}
a & b \\
c & -a \\
0 & 0
\end{array}\right)\left(\begin{array}{l}
\omega_{1} \\
\omega_{2}
\end{array}\right)
$$

Make a new manifold $M^{\prime}:=M \times \mathbb{R}_{a, b, c}^{3}$, and on $M^{\prime}$ let $\mathcal{I}^{\prime}$ be the exterior differential system generated by

$$
\left(\begin{array}{l}
\vartheta_{4} \\
\vartheta_{5} \\
\vartheta_{6}
\end{array}\right):=\left(\begin{array}{c}
\bar{\gamma}_{1} \\
\bar{\gamma}_{2} \\
\bar{\gamma}_{3}-\gamma
\end{array}\right)-\left(\begin{array}{cc}
a & b \\
c & -a \\
0 & 0
\end{array}\right)\left(\begin{array}{l}
\omega_{1} \\
\omega_{2}
\end{array}\right)
$$

9. Prolongation. Take an exterior differential system $\mathcal{I}$ on a manifold $M$. What should we do if there are no involutive integral elements? Let $M^{\prime}$ be the set of all pairs $(m, E)$ consisting of a point $m$ of $M$ and an $\mathcal{I}$-integral element $E \subset T_{m} M$. So $M^{\prime}$ is a subset of the Grassmann bundle over $M$. Locally on $M$, take a local basis $\omega, \vartheta, \pi$ of the 1 -forms, with $\vartheta$ a basis for the 1 -forms in $\mathcal{I}$. We can write each integral element on which $\omega$ has linearly independent components as the solutions of the linear equations $0=\vartheta, \pi=a \omega$ for some constants $a$. On an open subset of $M^{\prime}, a$ is a function valued in some vector space. Pull back the 1 -forms $\vartheta, \omega, \pi$ to $M^{\prime}$ via the $\operatorname{map}(m, E) \in M^{\prime} \mapsto m \in M$. On $M^{\prime}$, let $\vartheta^{\prime}:=\pi-a \omega$. The exterior differential system $\mathcal{I}^{\prime}$ on $M^{\prime}$ generated by $\vartheta^{\prime}$ is the prolongation of $\mathcal{I}$. Inductively, let $M^{(1)}:=M^{\prime}, \mathcal{I}^{(1)}:=\mathcal{I}^{\prime}, M^{(k+1)}:=\left(M^{(k)}\right)^{\prime}, \mathcal{I}^{(k+1)}:=\left(\mathcal{I}^{(k)}\right)^{\prime}$, if defined. 
ThEOREM 3 (Cartan-Kuranishi). If each $M^{(k)}$ is a submanifold of the Grassmann bundle over $M^{(k-1)}$, with finitely many connected components, and if each $M^{(k)} \rightarrow M^{(k-1)}$ is a submersion, then all but finitely many $\mathcal{I}^{(k)}$ are involutive.

10. Back to isometric immersion. Returning to our example of isometric immersion of surfaces, we have prolongation given by

$$
\left(\begin{array}{l}
\vartheta_{4} \\
\vartheta_{5} \\
\vartheta_{6}
\end{array}\right):=\left(\begin{array}{c}
\bar{\gamma}_{1} \\
\bar{\gamma}_{2} \\
\bar{\gamma}_{3}-\gamma
\end{array}\right)-\left(\begin{array}{cc}
a & b \\
c & -a \\
0 & 0
\end{array}\right)\left(\begin{array}{l}
\omega_{1} \\
\omega_{2}
\end{array}\right) .
$$

Note that $0=d \vartheta_{1}, d \vartheta_{2}, d \vartheta_{3}$ modulo $\vartheta_{4}, \vartheta_{5}, \vartheta_{6}$, so we can forget about them.

Calculate the exterior derivatives:

$$
d\left(\begin{array}{l}
\vartheta_{4} \\
\vartheta_{5} \\
\vartheta_{6}
\end{array}\right)=-\left(\begin{array}{cc}
D a & D b \\
D c & -D a \\
0 & 0
\end{array}\right) \wedge\left(\begin{array}{l}
\omega_{1} \\
\omega_{2}
\end{array}\right)+\left(\begin{array}{c}
0 \\
0 \\
t \omega_{1} \wedge \omega_{2}
\end{array}\right) \bmod \vartheta_{1}, \ldots, \vartheta_{6} .
$$

where

$$
\left(\begin{array}{c}
D a \\
D b \\
D c
\end{array}\right):=\left(\begin{array}{c}
d a+(b+c) \gamma-R_{23} \omega_{1} \\
d b-2 a \gamma+R_{13} \omega_{1} \\
d c-2 a \gamma
\end{array}\right)
$$

and the torsion is

$$
t:=\frac{s}{4}-R_{33}-K-a^{2}-b c .
$$

This torsion clearly has to vanish on any three-dimensional $\mathcal{I}^{\prime}$-integral element, i.e. every three-dimensional $\mathcal{I}^{\prime}$-integral element lives over the subset of $M^{\prime}$ on which

$$
0=\frac{s}{4}-R_{33}-K-a^{2}-b c .
$$

To ensure that this subset is a submanifold, we let $M_{0}^{\prime} \subset M^{\prime}$ be the set of points where this equation is satisfied and at least one of $a, b, c$ is not zero. Clearly $M_{0}^{\prime} \subset M^{\prime}$ is a submanifold, on which we find $D a, D b, D c$ linearly dependent. On $M_{0}^{\prime}$, every threedimensional $\mathcal{I}^{\prime}$-integral element on which $\omega_{1}, \omega_{2}, \gamma$ are linearly independent has $s_{1}=2$, $s_{2}=0$ and two dimensions of integral elements at each point. Therefore the exterior differential system is in involution: there is an integral manifold through each point of $M_{0}^{\prime}$, and in particular above every point of the surface. The prolongation exposes the hidden necessary condition for existence of a solution: the relation $t=0$ between the curvature of the ambient space, that of the surface, and the shape operator.

We will not prove the elementary:

Lemma 1. Every smooth three-dimensional integral manifold $X$ of the linear Pfaffian system constructed above on which $0 \neq \omega_{1} \wedge \omega_{2} \wedge \gamma$ is locally the adapted frame bundle of an isometric immersion open $\subset P \rightarrow \bar{P}$.

To sum up:

TheOREM 4. Take any surface $P$ with real analytic Riemannian metric, with chosen point $p_{0} \in P$ and Gauss curvature $K$. Take any 3-manifold $\bar{P}$ with real analytic Riemannian metric, with chosen point $\bar{p}_{0}$, and a linear isometric injection $F: T_{p_{0}} P \rightarrow T_{\bar{p}_{0}} \bar{P}$. Let $\nu$ be 
a unit normal vector to the image of $F$. Let $R$ be the Ricci tensor on that 3-manifold and $s$ the scalar curvature. Pick a nonzero quadratic form $q$ on the tangent plane $T_{p_{0}} P$ so that

$$
\operatorname{det} q=K+R(\nu, \nu)-\frac{s}{4}
$$

Then there is a real analytic isometric immersion $f$ of some neighborhood of $p_{0}$ to $\bar{P}$, so that $f^{\prime}\left(p_{0}\right)=F$ and so that $f$ induces shape operator $q$ at $p_{0}$.

11. For further information. For proof of the Cartan-Kähler theorem see [2, which we followed very closely, and also the canonical reference work [1] and the canonical textbook [6]. The last two also give proof of the Cartan-Kuranishi theorem. For more on triply orthogonal webs in Euclidean space, and orthogonal webs in Euclidean spaces of all dimensions, see [3, 4, 7, 8]. For more on isometric immersions and embeddings see [5].

\section{References}

[1] R. L. Bryant, S. S. Chern, R. B. Gardner, H. L. Goldschmidt, P. A. Griffiths, Exterior Differential Systems, Math. Sci. Res. Inst. Publ. 18, Springer, New York, 1991.

[2] É. Cartan, Les systèmes différentiels extérieurs et leurs applications géométriques, Actualités Sci. Ind., no. 994, Hermann et Cie., Paris, 1945.

[3] G. Darboux, Leçons sur les systèmes orthogonaux et les coordonnées curvilignes. Principes de géométrie analytique. The first title is a reprint of the second (1910) edition; the second title is a reprint of the 1917 original. Les Grands Classiques Gauthier-Villars, Éditions Jacques Gabay, Sceaux, 1993.

[4] D. M. DeTurck, D. Yang, Existence of elastic deformations with prescribed principal strains and triply orthogonal systems, Duke Math. J. 51 (1984), 243-260.

[5] Q. Han, J.-X. Hong, Isometric Embedding of Riemannian Manifolds in Euclidean Spaces, Math. Surveys Monogr. 130, Amer. Math. Soc., Providence, RI, 2006.

[6] T. A. Ivey, J. M. Landsberg, Cartan for Beginners: Differential Geometry via Moving Frames and Exterior Differential Systems, Grad. Stud. Math. 61, Amer. Math. Soc., Providence, RI, 2003.

[7] C. L. Terng, K. Uhlenbeck (eds.), Surveys in Differential Geometry: Integral Systems [Integrable Systems], a supplement to the J. Differential Geom., Surv. Diff. Geom. 4, International Press, Boston, MA, 1998.

[8] V. E. Zakharov, Description of the n-orthogonal curvilinear coordinate systems and Hamiltonian integrable systems of hydrodynamic type. I. Integration of the Lamé equations, Duke Math. J. 94 (1998), 103-139. 
